\title{
108. Effects of Inhibitors of Calcium-Induced Calcium Release on Receptor-Mediated Responses of Smooth Muscles and Platelets ${ }^{\dagger}$
}

\author{
By Shinobu YAgI,*),***) Noriko Matsumura,**) and Makoto Endo**) \\ (Communicated by Setsuro EBASHI, M. J. A., Oct. 14, 1985)
}

Ca-induced Ca release (CICR) first found in the sarcoplasmic reticulum of skeletal muscle ${ }^{1,2)}$ is an interesting biological phenomenon, but it does not appear to play a major role in normal excitation-contraction coupling in the amphibian skeletal muscle. ${ }^{3), 4)}$ However, it might play an important physiological role in other kinds of cells such as medaka eggs, as suggested by propagation of 'calcium wave' upon their fertilization. ${ }^{5}$ ) If a response of some cells is mediated by the CICR mechanism, it should be inhibited by inhibitors of CICR. Therefore, to examine whether or not inhibitors of CICR inhibit the response constitutes a good screening test for the possibility of involvement of the CICR mechanism in evoking the response in question. Indeed, the absence of inhibition of normal excitation-contraction coupling of amphibian skeletal muscle by inhibitors of $\mathrm{CICR}^{6), 7)}$ furnished strong evidence against physiological role of the mechanism in this tissue. In the present article, the effect of procaine and adenine, two different inhibitors of CICR, ${ }^{6), 8), 9)}$ was examined on contractions of smooth muscles evoked by transmitters and on shape changes of platelets.

Thin bundles of smooth muscle fibers were dissected from taenia caeci or portal vein from the guinea pig. They were suspended in a trough in which solutions could be exchanged rapidly. Isometric tension was monitored by a strain-gauge transducer (UL-2, Shinkoh, Tokyo) and displayed on a pen recorder. Contractions were evoked by applying caffeine or neurotransmitters, acetylcholine or carbachol in taenia caeci and noradrenaline in portal vein. To see the result of Ca release from an intracellular store without participation of changes in membrane potential and of Ca entry from the extracellular fluid, experiments were done in a medium in which all $\mathrm{Na}$ ions were replaced by $\mathrm{K}$ ions and $4 \mathrm{mM}$ EGTA (ethyleneglycolbis-( $\beta$-aminoethylether)- $\mathrm{N}-\mathrm{N}^{\prime}$-tetraacetic acid) was present.

Platelets were obtained from arterial blood drawn through a plastic cannula inserted into the femoral artery of albino rabbits under ether anesthesia. Blood was immediately mixed with $1 / 9$ volume of sodium citrate solution (final concentration about $11 \mathrm{mM}$ ) and platelet-rich plasma obtained as the supernatant by centrifuging the citrated blood at $230 \times \mathrm{g}$ for $7 \mathrm{~min}$. One volume of the platelet-rich plasma was mixed with 4 volume of a buffer $\left(\mathrm{Na}_{2} \mathrm{SO}_{4}\right.$ or $\mathrm{K}_{2} \mathrm{SO}_{4}$

†) Supported partly by a Grant-in-Aid for Scientific Research from the Ministry of Education, Science and Culture, Japan and by Grant from the Ministry of Health and Welfare, Japan.

*) Department of Pharmacology, Tohoku University School of Medicine, Sendai 980 .

**) Department of Pharmacology, Faculty of Medicine, University of Tokyo, Bunkyo-ku, Tokyo 113.

***) Present address: Department of Physiology, University of Massachusetts Medical Center, Worcester, Massachusetts 01605, U.S.A. 
$90 \mathrm{mM}, \mathrm{MgSO}_{4} 10 \mathrm{mM}$, EGTA $5 \mathrm{mM}$, HEPES (N-2-hydroxyethylpiperazine-N'-2ethanesulfonic acid) $5 \mathrm{mM}$ at $\mathrm{pH} 7.4$, glucose $5.5 \mathrm{mM}$ ) and its turbidity change on an application of $1 / 20$ or $1 / 40$ volume of concentrated stimulating agents was monitored at $37^{\circ} \mathrm{C}$ by an aggregometer (NKK Hema Tracer PAT $4 \mathrm{M}$, Niko Bioscience, Tokyo).

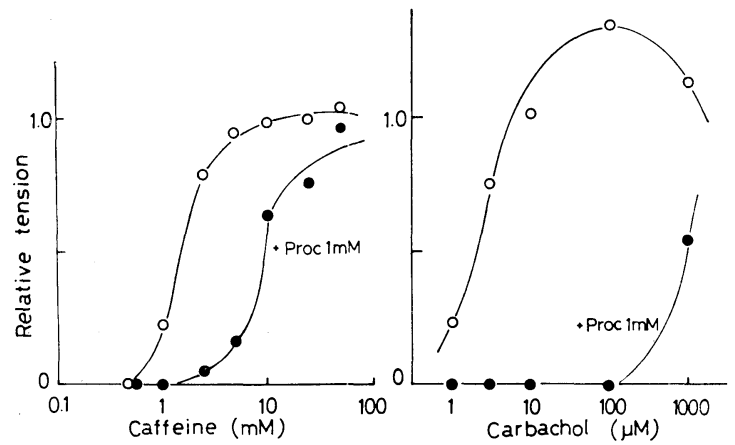

Fig. 1. The effect of procaine on caffeine- and carbachol contractions of a thin fiber bundle from taenia caeci of the guinea pig in $4 \mathrm{mM}$ EGTAcontaining $\mathrm{K}$ solution. Between two successive contractions, the bundle was treated with a solution containing $2 \mathrm{mM}$ Ca for 5 min to 'load' Ca. ${ }^{19}$ The size of peak tension relative to that with $25 \mathrm{mM}$ caffeine without procaine was plotted. Open circles, without procaine; closed circles with $1 \mathrm{mM}$ procaine.

Fig. 1 shows the effect of procaine, an inhibitor of CICR, on caffeine- and carbachol-induced contraction of taenia caeci. As expected and as already reported,10) it inhibited caffeine contracture that was probably a result of enhanced CICR. ${ }^{11)}$ Carbachol contracture was also inhibited by procaine even more strongly. Almost the same result was obtained with adenine, another inhibitor of CICR, on caffeine- and noradrenaline-contraction of portal vein as Endo and Kitazawa reported. ${ }^{12)}$ The same was true for the effect of adenine on taenia caeci and for that of procaine on portal vein. On the other hand, procaine was reported not to alter the relationship between $\mathrm{Ca}$ ion concentration and tension in skinned smooth muscle fibers appreciably. ${ }^{10)}$ It was found that the same was true for adenine (data not shown).

In platelets, upon addition of stimulating agents such as A23187 or thrombin, an increase in turbidity of the platelet suspension due to their shape change ${ }^{13}$ ) followed an immediate decrease in turbidity that was a result of dilution of platelets by the addition of the solution of the stimulating agents (Fig. 2). Aggregation did not occur in our experiments except at very high concentrations of thrombin (data not shown), because Ca ions were essentially absent in the extracellular medium. Fig. 2 shows that procaine up to $10 \mathrm{mM}$ did not inhibit the shape change of platelets induced by $250 \mathrm{nM}$ of A23187. Thus, Ca ion released from the internal store by the ionophore can cause similar responses in the presence of procaine. However, the drug clearly inhibited thrombin-induced shape change of the platelets. Fig. 3 shows dose-response curves of A23187 and thrombin under various concentrations of adenine. On the ordinate, maximum rate of turbidity increase was plotted as an index of the magnitude of the 

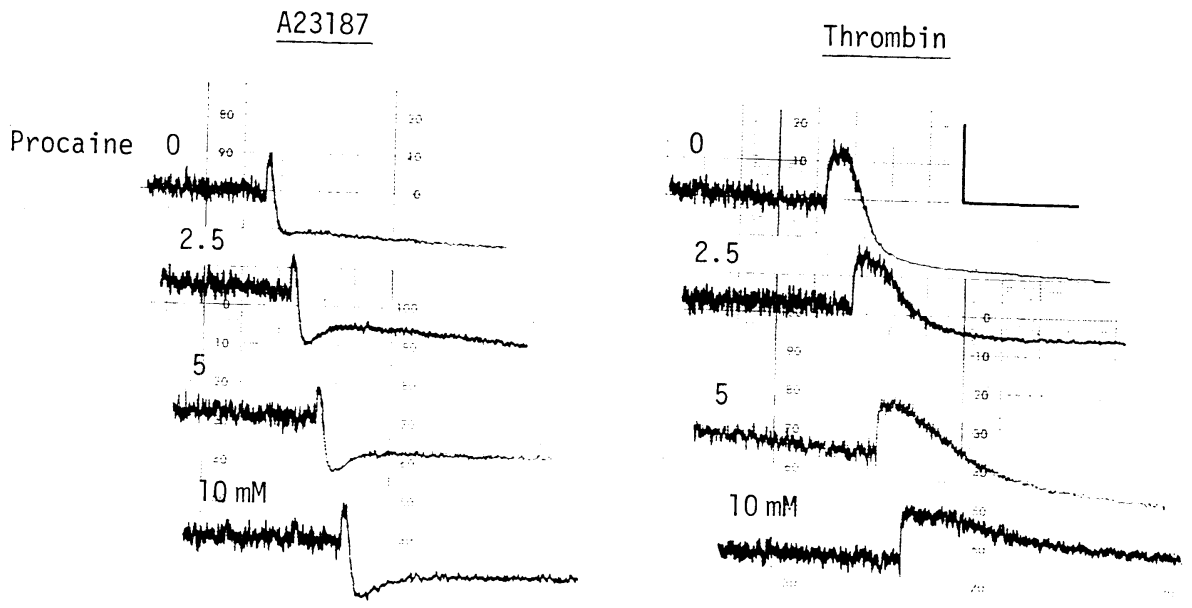

Fig. 2. The effect of procaine on an increase in turbidity of suspensions of rabbit platelets induced by $250 \mathrm{nM} \mathrm{A} 23187$ (left panel) and $0.75 \mathrm{unit} / \mathrm{ml}$ thrombin (right panel). The stimulating agents were added at the time of abrupt upward shift of the turbidity. Turbidity increase downward. Bars indicate $10 \%$ and $1 \mathrm{~min}$.
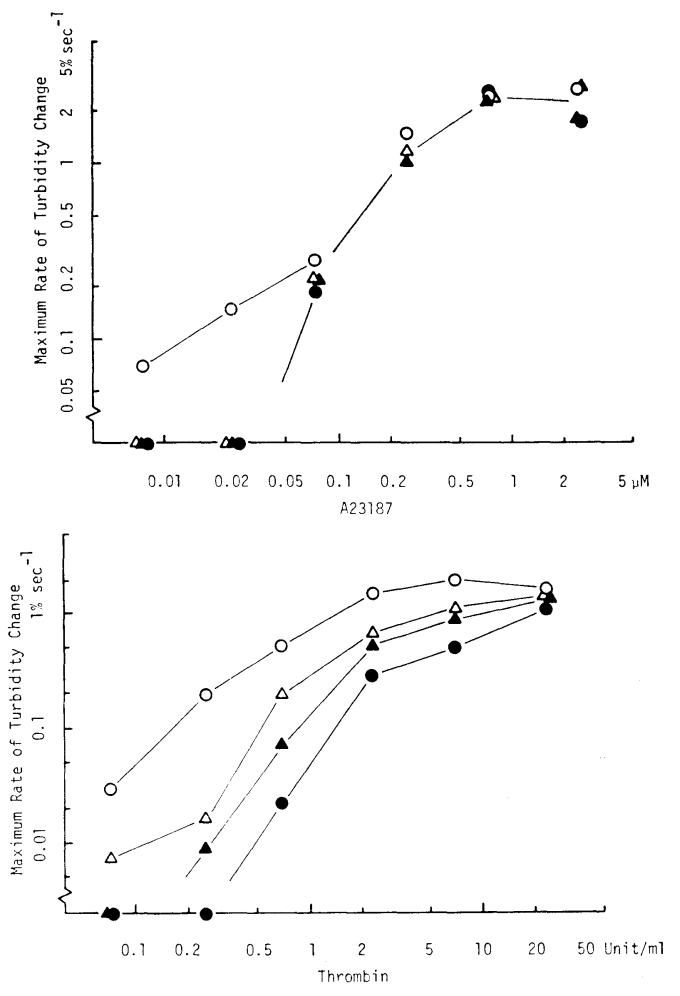

Fig. 3. The effect of adenine on the maximum rate of turbidity increase of rabbit platelet suspensions induced by A23187 and thrombin. The rate was measured on such a turbidity tracing as shown in Fig. 2 .

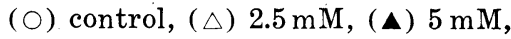
(•) $10 \mathrm{mM}$ adenine. 
[Vol. 61(B),

response. It is clear that while adenine did not appreciably inhibit responses induced by A23187 except at its lowest concentrations, it inhibited thrombininduced responses at all the concentrations of thrombin used. Very similar families of dose-response curves were obtained with procaine in place of adenine.

Thus, both procaine and adenine, two different inhibitors of CICR, inhibited receptor-mediated responses due to $\mathrm{Ca}$ release from the intracellular $\mathrm{Ca}$ store in smooth muscles as well as in platelets. The inhibition was due to an inhibition of Ca release, because both inhibitors essentially do not inhibit responses caused by direct application of $\mathrm{Ca}$ ion. Therefore, unlike excitation-contraction coupling of amphibian skeletal muscle, the possibility that the CICR mechanism plays an important role in the receptor-mediated $\mathrm{Ca}$ release from an intracellular Ca store, of smooth muscles, of platelets and probably of other nonmuscle cells, remains to be an attractive working hypothesis. The physiological function of the CICR mechanism in smooth muscle cells has already been proposed on similar or other grounds.10),12),14),15) $\mathrm{Ca}$ ion might be the mediator released from somewhere by agent-receptor interactions to cause further release of Ca from the major Ca store. Or, the CICR mechanism might play an important role as the amplifying mechanism, some other substance being the triggering mediator. At present the possibility cannot be excluded that the inhibitory effect of procaine and adenine reported here is a result of their actions unrelated to the CICR inhibition. Furthermore, it is still to be shown whether there really is a CICR mechanism in the $\mathrm{Ca}$ store of platelets. Nevertheless, in order to elucidate the mechanism of receptor-mediated responses of smooth muscle and nonmuscle cells, further studies along the working hypothesis stated above seem to be very much worth doing. Another attractive hypothesis recently proposed is that inositol trisphosphate $\left(\mathrm{IP}_{3}\right)$ may be the mediator for the coupling between the agentreceptor interaction and $\mathrm{Ca}$ release from the $\mathrm{Ca}$ store.16)-18) However, while $\mathrm{IP}_{3}$-induced $\mathrm{Ca}$ release from the smooth muscle $\mathrm{Ca}$ store is not inhibited by procaine (M. Iino, K. Horiuti and M. Endo, unpublished result), receptor-mediated responses were very effectively inhibited by procaine as shown here. Therefore, $\mathrm{IP}_{3}$ is rather unlikely to be the only mediator in smooth muscles, unless procaine inhibits $\mathrm{IP}_{3}$ formation.

\section{References}

1) Ford, L. E., and Podolsky, R. J.: Science, 167, 58-59 (1970).

2) Endo, M. et al.: Nature, 228, 34-36 (1970).

3) Endo, M.: Physiol. Rev., 57, 71-108 (1977).

4) : Jikeikai Med. J., 30, 123-130 (1984).

5) Ridgway, E. B. et al.: ' Proc. Natl. Acad. Sci. U.S.A., 74, 623-627 (1977).

6) Thorens, S., and Endo, M.: Proc. Japan Acad., 51, 473-478 (1975).

7) Ishizuka, T. et al.: ibid., 59B, 97-100 (1983).

8) Ford, L. E., and Podolsky, R. J.: J. Physiol., 223, 1-19 (1972).

9) Ishizuka, T., and Endo, M.: Proc. Japan Acad., 59B, 93-96 (1983).

10) Itoh, T. et al.: J. Physiol., 321, 513-525 (1981).

11) Endo, M.: Proc. Japan Acad., 51, 479-484 (1975).

12) Endo, M., and Kitazawa, T.: Calcium Entry Blockers and Tissue Protection (ed. T. Godfraind et al.). Raven Press, New York, pp. 81-89 (1985).

13) Born, G. V. R.: J. Physiol., 209, 487-511 (1970).

14) Saida, K.: J. Gen. Physiol., 80, 191-202 (1982).

15) Saida, K., and van Breemen, C.: Pflügers Arch., 397, 166-167 (1983).

16) Streb, H. et al.: Nature, 306, 67-69 (1983).

17) Suematsu, E. et al.: Biochem. Biophys. Res. Commun., 120, 481-485 (1984).

18) Somlyo, A. V. et al.: Proc. Natl. Acad. Sci. U.S.A. (in press).

19) Endo, M.: Muscle Contraction: Its Regulatory Mechanisms (ed. S. Ebashi et al.). Japan Sci. Soc. Press, Tokyo, pp. 447-463 (1980). 